\title{
Semiexclusive production of vector mesons in proton-proton collisions with electromagnetic dissociation
}

\author{
Anna Cisek, ${ }^{1, *}$ Wolfgang Schäfer ${ }^{2, \dagger}$ and Antoni Szczurek $\odot^{2,1, \$}$ \\ ${ }^{1}$ College of Natural Sciences, Institute of Physics, University of Rzeszów, \\ ul. Pigonia 1, PL-35-310 Rzeszów, Poland \\ ${ }^{2}$ Institute of Nuclear Physics, Polish Academy of Sciences, \\ ul. Radzikowskiego 152, PL-31-342 Kraków, Poland
}

(Received 17 October 2019; published 12 December 2019)

\begin{abstract}
We calculate distributions of different vector mesons in purely exclusive $(p p \rightarrow p p V)$ and semiexclusive $(p p \rightarrow p X V)$ processes with the electromagnetic dissociation of a proton. The cross section for the electromagnetic dissociation is expressed through electromagnetic structure functions of the proton. We include the transverse momentum distribution of initial photons in the associated flux. Contributions of the exclusive and semiexclusive processes are compared for different vector mesons $(V=\phi, J / \psi, \Upsilon)$. We discuss how the relative contribution of the semiexclusive processes depends on the mass of the vector meson as well as on different kinematical variables of the vector meson $\left(y, p_{t}\right)$. The ratio of semiexclusive to exclusive contributions are shown and compared for different mesons in different variables.
\end{abstract}

DOI: $10.1103 /$ PhysRevD.100.114022

\section{INTRODUCTION}

The exclusive production of vector mesons $p p \rightarrow p p V$ is a source of knowledge about gluon distributions in the proton. In contrast to the collinear approach, in the $k_{t}$-factorization approach, the cross section depends not only on the (unintegrated) gluon distribution function (UGDF) but also on the quarkonium quark-antiquark wave function [1]. It was shown that different UGDFs give different results. In order to "extract" or check the collinear gluon distribution or UGDF, one has to be sure that the measured cross sections are not contaminated by any other mechanism.

So far, both $J / \psi$ and $\Upsilon[2,3]$ were measured in protonproton collisions. The measurements are not fully exclusive as so far, the outgoing protons were not measured. To increase the exclusivity of the reaction, a rapidity veto (no emission around the rapidity of a vector meson) is being imposed. How good is such an approach is not fully understood in our opinion. In our earlier paper on $J / \psi$ production [4], we have developed a formalism how to calculate such processes with rapidity gaps, but including proton dissociation. To calculate electromagnetic

\footnotetext{
*acisek@ur.edu.pl

†olfgang.Schafer@ifj.edu.pl

¥Antoni.Szczurek@ifj.edu.pl
}

Published by the American Physical Society under the terms of the Creative Commons Attribution 4.0 International license. Further distribution of this work must maintain attribution to the author(s) and the published article's title, journal citation, and DOI. Funded by SCOAP. dissociation, the method uses parametrizations of the proton structure functions which are used to derive an inelastic photon flux. We have shown in [4] that the semiexclusive mechanism cannot be completely removed by the requirement of rapidity veto. To our surprise, the electromagnetic dissociation seems the most important (the largest) in this context. Here we wish to show more systematic studies for the production of different vector mesons and better understand the competition of the purely exclusive and the semiexclusive processes.

The semiexclusive production mechanisms are shown for illustration in Fig. 1. Here, due to the quantum numbers of vector mesons, the dominant mechanism is photonpomeron fusion. As shown in the figure, the photon can be coupled to either one of the two protons.

In this work, we will calculate different differential distributions. Of special interest is the ratio of the semiexclusive to exclusive cross section. Such a ratio may be considered as a measure of "unwanted" contamination of exclusive processes when using the rapidity gap method. The predictions of cross section for the semiexclusive processes may be also valuable as it could be in principle measured.

\section{SKETCH OF THE FORMALISM}

Let us concentrate on the events with the electromagnetic dissociation of one of the protons. The important property of these processes is that the $p \gamma^{*} \rightarrow X$ transition is given by the electromagnetic structure functions of the proton and, thus to a large extent, calculable "from data". The cross section for such processes can be written as 


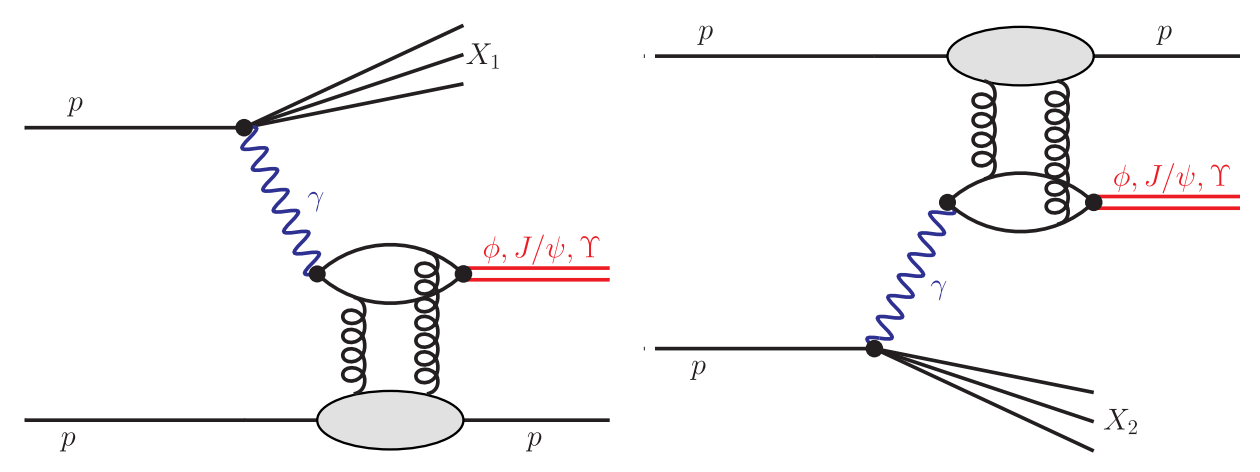

FIG. 1. Schematic representation of the electromagnetic excitation of one (left panel) or second (right panel) proton.

$$
\frac{d \sigma(p p \rightarrow X V p ; s)}{d y d^{2} \mathbf{p} d M_{X}^{2}}=\int \frac{d^{2} \mathbf{q}}{\pi \mathbf{q}^{2}} \mathcal{F}_{\gamma / p}^{(\mathrm{inel})}\left(z_{+}, \mathbf{q}^{2}, M_{X}^{2}\right) \frac{1}{\pi} \frac{d \sigma^{\gamma^{*} p \rightarrow V p}}{d t}\left(z_{+} s, t=-(\mathbf{q}-\mathbf{p})^{2}\right)+\left(z_{+} \leftrightarrow z_{-}\right)
$$

where $z_{ \pm}=e^{ \pm y} \sqrt{\left(\mathbf{p}^{2}+m_{V}^{2}\right) / s}$ is the fraction of the proton's longitudinal momentum carried by the photon, and $M_{X}$ is the invariant mass of the excited system $X, \mathbf{p}$ is the transverse momentum of the vector meson, and $-\mathbf{q}$ is the transverse momentum of the outgoing hadronic system $X$. Below we also use $p_{t}=|\mathbf{p}|$ for the absolute value of the transverse momentum. The mass of the excited hadronic system must be above the threshold $M_{\mathrm{thr}}=m_{\pi}+m_{p}$. In the kinematics of interest, the "fully unintegrated" flux of photons associated with the breakup of the proton is calculable in terms of the structure function $F_{2}$ of a proton,

$$
\mathcal{F}_{\gamma / p}^{(\text {inel })}\left(z, \mathbf{q}^{2}, M_{X}^{2}\right)=\frac{\alpha_{\mathrm{em}}}{\pi}(1-z) \theta\left(M_{X}^{2}-M_{\mathrm{thr}}^{2}\right) \frac{F_{2}\left(x_{B j}, Q^{2}\right)}{M_{X}^{2}+Q^{2}-m_{p}^{2}}\left[\frac{\mathbf{q}^{2}}{\mathbf{q}^{2}+z\left(M_{X}^{2}-m_{p}^{2}\right)+z^{2} m_{p}^{2}}\right]^{2},
$$

where we calculate the photon virtuality $Q^{2}$ and the Bjorken variable $x_{\mathrm{Bj}}$ from

$$
\begin{aligned}
Q^{2} & =\frac{1}{1-z}\left[\mathbf{q}^{2}+z\left(M_{X}^{2}-m_{p}^{2}\right)+z^{2} m_{p}^{2}\right], \\
x_{B j} & =\frac{Q^{2}}{Q^{2}+M_{X}^{2}-m_{p}^{2}} .
\end{aligned}
$$

We use the following parametrizations of the proton structure function $F_{2}\left(x, Q^{2}\right)$ :

(1) A parametrization of Refs. [5,6], which is fitted to the lower energy CLAS ${ }^{1}$ data and is meant to give an accurate description, especially in the resonance region $M_{X} \lesssim 2 \mathrm{GeV}$. In the figures, it will be labeled as FFJLM. This parametrization does not describe data well when it is extrapolated beyond the region of its intended use. Therefore, we only use it when calculating observables with $M_{X} \lesssim 2 \mathrm{GeV}$.

(2) The Abramowicz-Levy-Levin-Maor fit [7,8] also used previously in [9], abbreviated here ALLM.

(3) A newly constructed parametrization, which at $Q^{2}>9 \mathrm{GeV}^{2}$ uses an next-to-next-to-leading-order (NNLO) calculation of $F_{2}$ and $F_{L}$ from NNLO

${ }^{1} \mathrm{CLAS}=\mathrm{CEBAF}$ large acceptance spectrometer (where CEBAF is continuous electron beam accelerator facility).
MSTW 2008 partons [10]. It employs a useful code by the MSTW group [10] to calculate structure functions. At $Q^{2}>9 \mathrm{GeV}^{2}$, this fit uses the parametrization of Bosted and Christy [11] in the resonance region and a version of the ALLM fit published by the HERMES Collaboration [12] for the continuum region. It also uses information on the longitudinal structure function from SLAC [13]. As

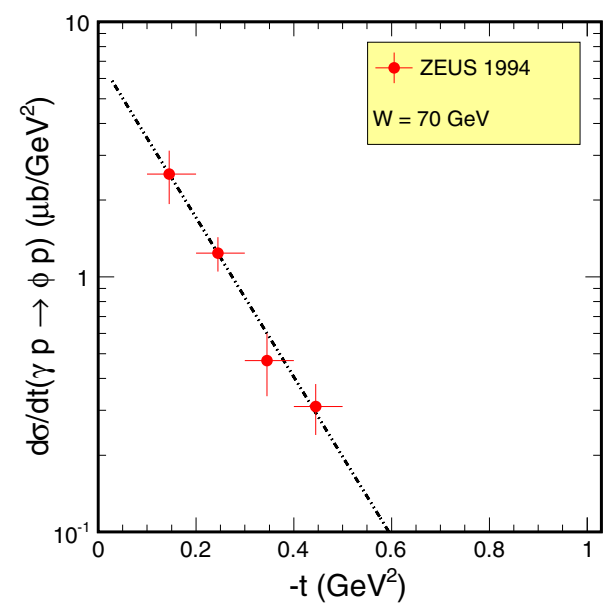

FIG. 2. Differential cross section for diffractive photoproduction of $\phi$ mesons. Data are from the ZEUS Collaboration at HERA [23]. 

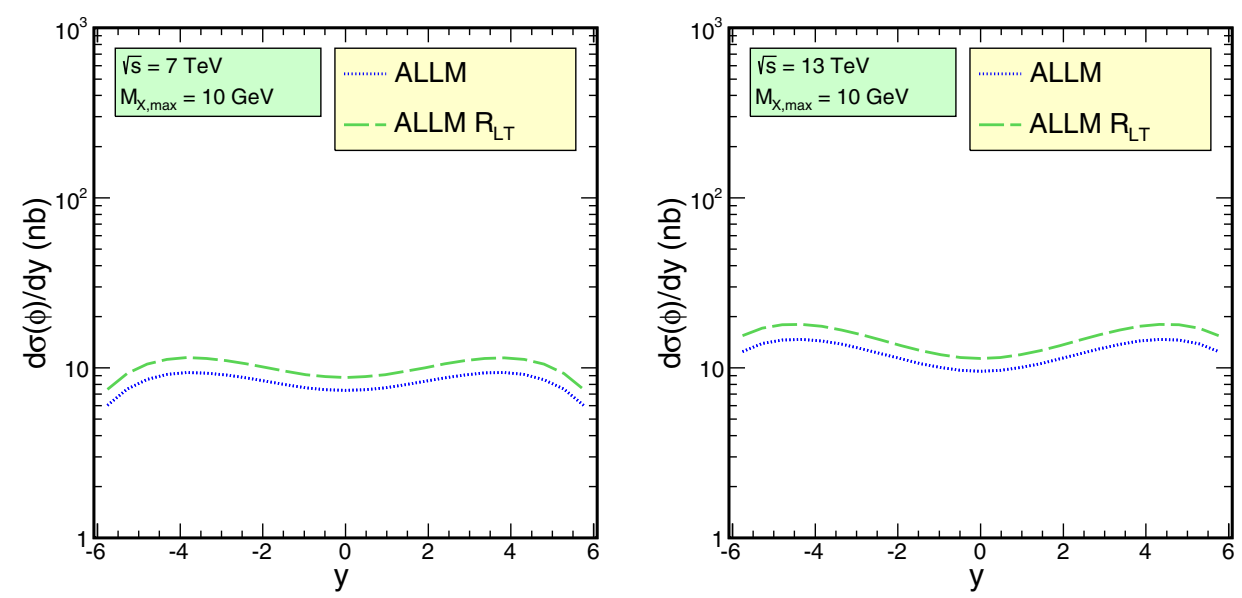

FIG. 3. Rapidity distribution of $\phi$ mesons at two different energies, with and without the contribution from longitudinal photons.

the fit is constructed closely following the LUXqed work Refs. [14,15], we call this fit LUX-like.

(4) A vector-meson-dominance model inspired fit of $F_{2}$ proposed in [16] at low $Q^{2}$, which is completed by the same NNLO MSTW structure function as above at large $Q^{2}$. This fit is labeled SU for brevity.

Our formalism is valid for photons which carry momentum fractions $z \ll 1$; this is an appropriate approximation for the production of vector mesons away from the forward rapidity regions. The differential cross section for the $\gamma^{*} p \rightarrow V p$ process is

$$
\begin{aligned}
\frac{d \sigma^{\gamma^{*} p \rightarrow V p}}{d t} & =\frac{d \sigma_{T}^{\gamma_{T}^{*} p \rightarrow V p}}{d t}+\frac{d \sigma_{L}^{\gamma_{L}^{*} p \rightarrow V p}}{d t} \\
& =\frac{d \sigma_{T}^{\gamma_{T}^{*} p \rightarrow V p}}{d t}\left(1+R_{L T}\left(Q^{2}\right)\right) .
\end{aligned}
$$

Although the focus of our work is on dissociative events, we also wish to compare some distributions to the ones of fully exclusive events. Let us briefly describe the theoretical formulation used for the exclusive $p p \rightarrow p p V$ process.

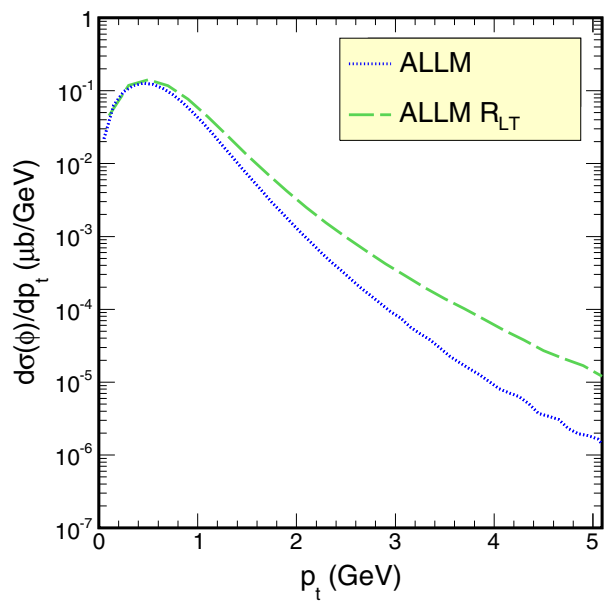

We concentrate on the photon-Pomeron fusion and neglect a possible Odderon-Pomeron fusion contribution, as well as other secondary exchanges. The amplitude for the diffractive exclusive process $\gamma^{*} p \rightarrow V p$ is written in the form,

$$
\begin{aligned}
& \mathcal{M}\left(\gamma^{*} p \rightarrow V p ; s, t, Q^{2}\right) \\
& \quad=(i+\rho) \Im m \mathcal{M}\left(\gamma^{*} p \rightarrow V p ; s, 0, Q^{2}\right) \exp \left[B_{V}(s) t / 2\right]
\end{aligned}
$$

For the imaginary part of the amplitude, there holds a $k_{T^{-}}$ factorization formula (see, e.g., the review in Ref. [17] and references therein; here we only include the $s$-channel helicity conserving amplitude and suppress helicity labels),

$$
\begin{aligned}
\Im & m \mathcal{M}\left(\gamma^{*} p \rightarrow V p ; s, 0, Q^{2}\right) \\
& =\int \frac{d^{2} \boldsymbol{\kappa}}{\boldsymbol{\kappa}^{4}} \mathcal{F}\left(x, \boldsymbol{\kappa}^{2}\right) \int_{0}^{1} d z d^{2} \boldsymbol{k} I^{\gamma^{*} \rightarrow V}\left(z, \boldsymbol{k}, \boldsymbol{\kappa}, Q^{2}\right) \psi_{V}(z, \boldsymbol{k}),
\end{aligned}
$$

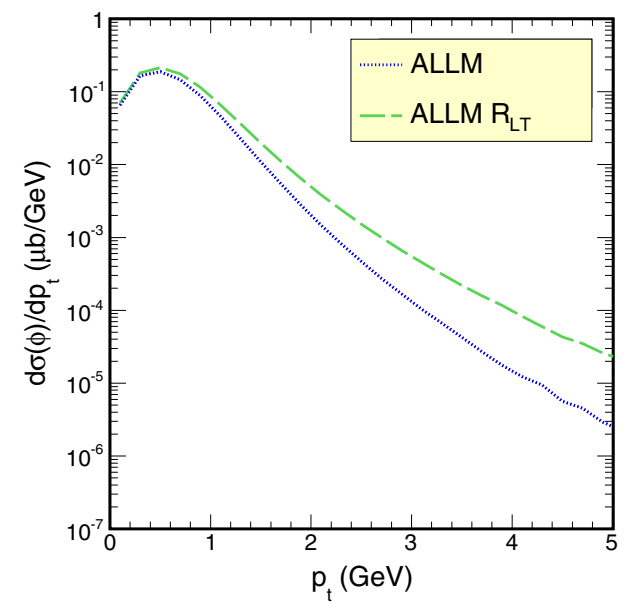

FIG. 4. Transverse momentum distribution of $\phi$ mesons at two different energies, with and without the contribution from longitudinal photons for energy $\sqrt{s}=7 \mathrm{TeV}$ (left panel) and $\sqrt{s}=13 \mathrm{TeV}$ (right panel). 

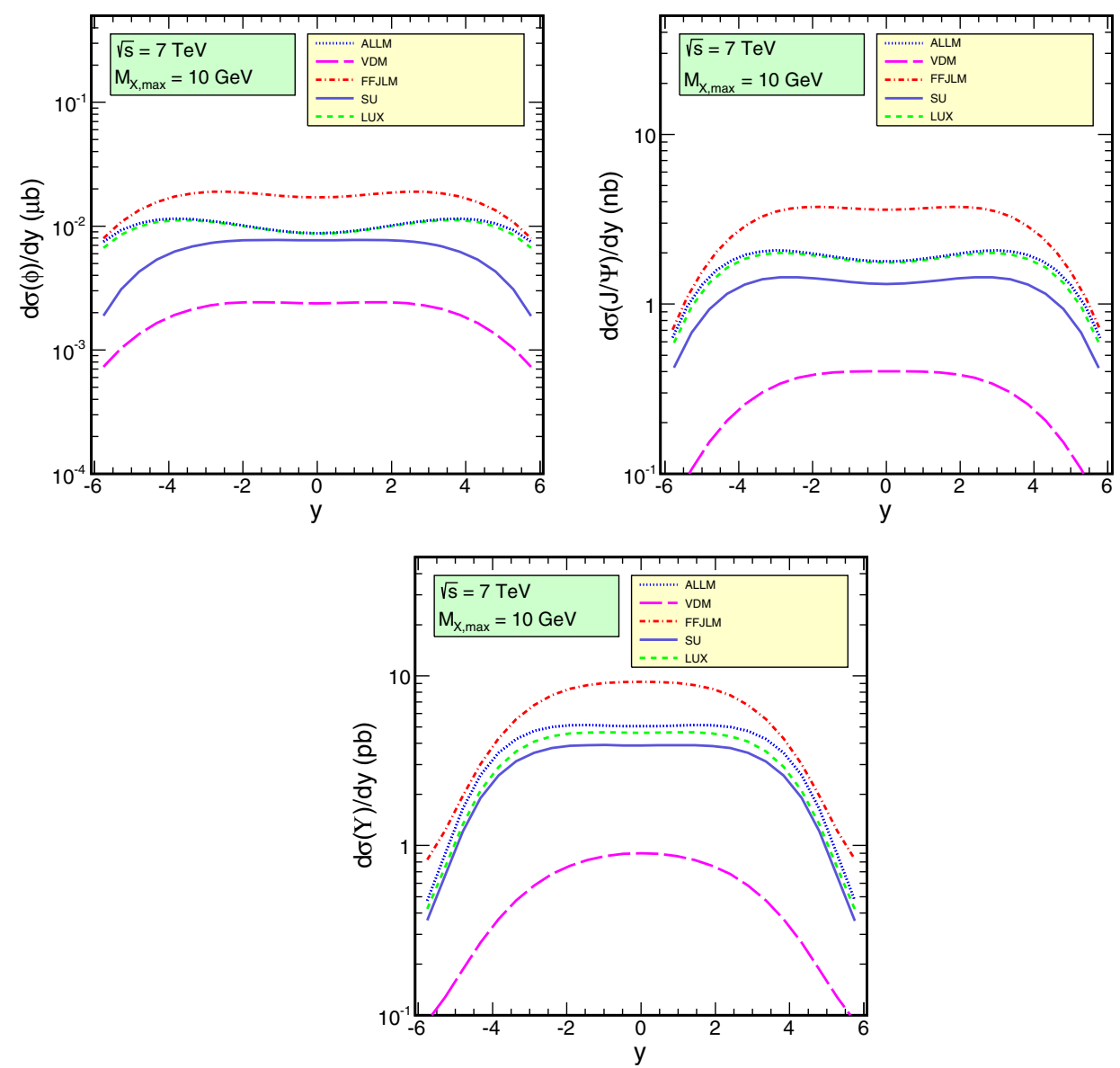

FIG. 5. Rapidity distribution for pp cm energy $\sqrt{s}=7 \mathrm{TeV}$ for the production of $\phi, J / \psi$, and $\Upsilon$ mesons for different parametrizations of the proton structure function $F_{2}$.

where $\mathcal{F}\left(x, \boldsymbol{\kappa}^{2}\right)$ is the unintegrated gluon distribution of the proton, and $\psi_{V}(z, \boldsymbol{k})$ is the $q \bar{q}$ light cone wave function of the meson. Here, $z$ is the fraction of the meson longitudinal momentum carried by the quark, and $\boldsymbol{k}$ is the quark transverse momentum. Details on the respective wave functions and explicit expressions used for $I^{\gamma^{*} \rightarrow V}$ can be found in $[1,18]$. We used an unintegrated gluon distribution from Ref. [19]. The real part of the diffractive amplitude is calculated from the relation,

$$
\begin{aligned}
\rho & =\frac{\Re e \mathcal{M}\left(\gamma^{*} p \rightarrow V p ; s, Q^{2}, 0\right)}{\Im m \mathcal{M}\left(\gamma^{*} p \rightarrow V p ; s, Q^{2}, 0\right)}=\tan \left(\frac{\pi \Delta_{\mathbf{I P}}}{2}\right), \\
\Delta_{\mathbf{I P}} & =\frac{\partial \log (\Im m \mathcal{M} / s)}{\partial \log s} .
\end{aligned}
$$

For the diffraction slope, we use

$$
B_{V}(s)=b_{V}+2 \alpha_{\mathrm{eff}}^{\prime} \log \left(\frac{s}{s_{0}}\right)
$$

with $\alpha_{\text {eff }}^{\prime}=0.164 \mathrm{GeV}^{-2}$, and $s_{0}=(95 \mathrm{GeV})^{2}$. We used $b_{\phi}=7.5 \mathrm{GeV}^{-2}, b_{J / \psi}=4.88 \mathrm{GeV}^{-2}$ and $b_{\Upsilon}=3.5 \mathrm{GeV}^{-2}$, respectively. Experimental data for $J / \psi$-production can be found in [20,21]. The formalism for the $2 \rightarrow 3$ exclusive process is described in detail in Refs. [1,22]. There are two interfering amplitudes, accounting for the fact that the photon can couple to either proton. The bare amplitude, without absorption effects has the form,

$$
\begin{aligned}
\mathcal{M}_{\lambda_{1} \lambda_{2} \rightarrow \lambda_{1}^{\prime} \lambda_{2}^{\prime} \lambda_{V}}^{p p \rightarrow p p V}= & \mathcal{M}_{\gamma \mathbf{I P}}+\mathcal{M}_{\mathbf{I P} \gamma} \\
= & \left\langle p_{1}^{\prime}, \lambda_{1}^{\prime}\left|J^{\mu}\right| p_{1} \lambda_{1}\right\rangle \varepsilon_{\mu}^{*}\left(q_{1}, \lambda_{V}\right) \frac{\sqrt{4 \pi \alpha_{\mathrm{em}}}}{t_{1}} \mathcal{M}\left(\gamma^{*} p \rightarrow V p ; s_{2}, t_{2}, Q_{1}^{2}\right) \\
& +\left\langle p_{2}^{\prime}, \lambda_{2}^{\prime}\left|J^{\mu}\right| p_{2} \lambda_{2}\right\rangle \varepsilon_{\mu}^{*}\left(q_{2}, \lambda_{V}\right) \frac{\sqrt{4 \pi \alpha_{\mathrm{em}}}}{t_{2}} \mathcal{M}\left(\gamma^{*} p \rightarrow V p ; s_{1}, t_{1}, Q_{2}^{2}\right) .
\end{aligned}
$$



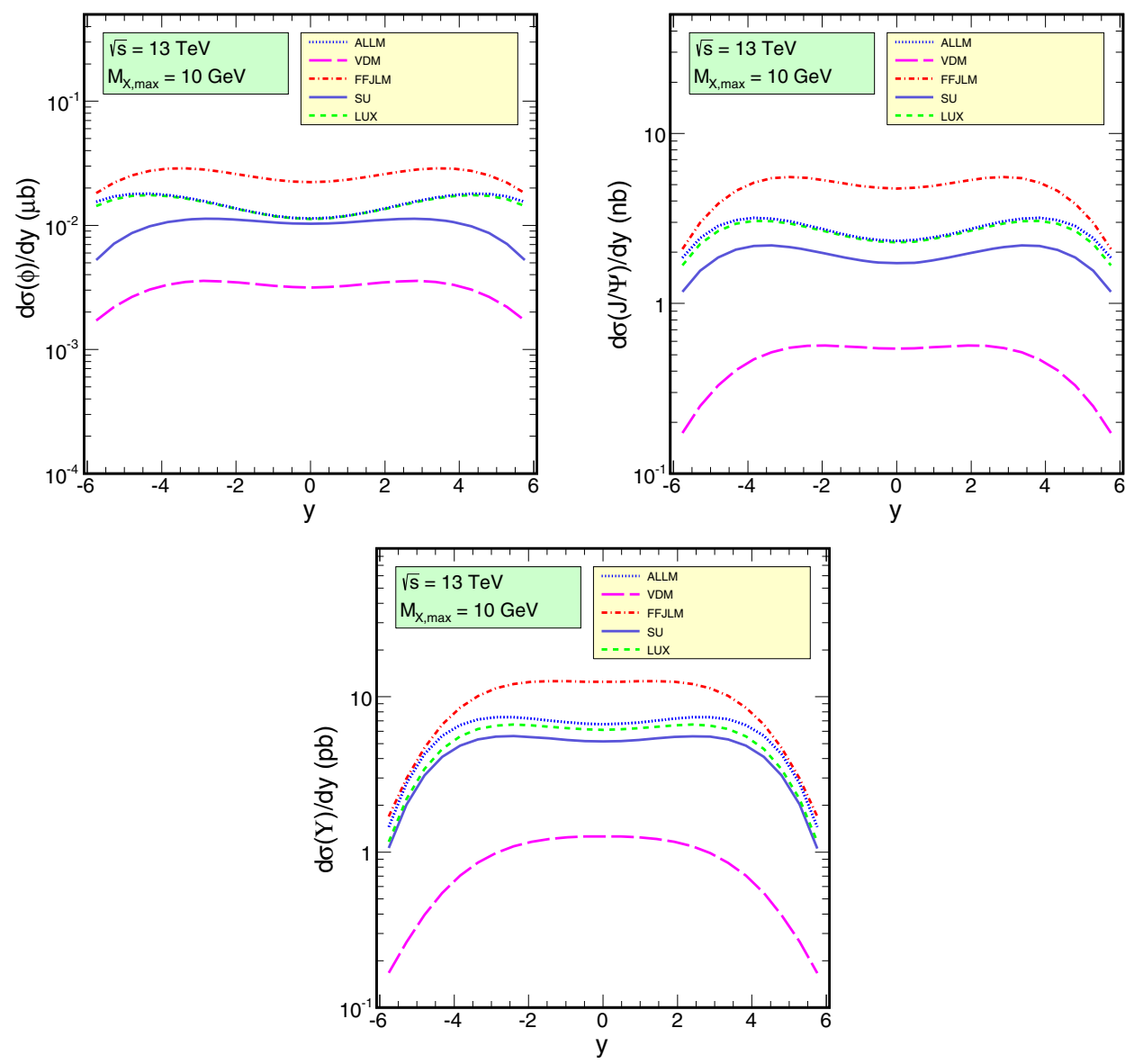

FIG. 6. Rapidity distribution for $\mathrm{pp} \mathrm{cm}$ energy $\sqrt{s}=13 \mathrm{TeV}$ for the production of $\phi, J / \psi$, and $\Upsilon$ mesons for different parametrizations of the proton structure function $F_{2}$.

The differential cross section is then obtained from

$$
d \sigma=\frac{1}{512 \pi^{4} s^{2}} \overline{\left|\mathcal{M}_{\lambda_{1} \lambda_{2} \rightarrow \lambda_{1}^{\prime} \lambda_{2}^{\prime} \lambda_{V}}^{p p \rightarrow p p V}\right|^{2}} d y d t_{1} d t_{2} d \phi
$$

where $y$ is the rapidity of the vector meson and $\phi$ is the azimuthal angle between outgoing protons. For the case of $J / \psi$, these results are similar to a parametrization used in [22].

In Fig. 2, we show as an example the differential cross section for $\phi$ photoproduction, comparing our simple fit with the data taken by the ZEUS Collaboration at HERA [23].

\section{RESULTS}

We start our discussion of results with the rapidityand transverse-momentum distributions of semiexclusively produced $\phi$ mesons. Here, we want to discuss the effect of longitudinal photons quantified by $R_{L T}$ in Eq. (2.4). In Fig. 3, we show the rapidity dependent cross section,

$$
\frac{d \sigma(\phi)}{d y} \equiv \int_{M_{\mathrm{thr}}}^{M_{X, \max }} d M_{X} \frac{d \sigma(p p \rightarrow \phi X ; s)}{d y d M_{X}},
$$

integrated up to masses $M_{X, \max }=10 \mathrm{GeV}$ with and without the $R_{L T}$ term included. We observe, that longitudinal photons enhance the cross section by about $20 \%$ uniformly in $y$. As we can see from the transverse-momentum distribution of $\phi$ mesons shown in Fig. 4, the effect of longitudinal photons is important at large transverse momenta, $p_{t}>1 \mathrm{GeV}$. For heavier mesons, we find small effects of longitudinal photons, as the ratio behaves like $R_{L T} \propto Q^{2} / m_{V}^{2}$, over a broad range of $Q^{2}$. This means a suppression of longitudinal photons in the relevant, for us, range of $Q^{2}$.

We now wish to present results for rapidity and transverse-momentum distributions of mesons. In Figs. 5 and 6, we show the rapidity dependent cross section,

$$
\frac{d \sigma(V)}{d y} \equiv \int_{M_{\mathrm{thr}}}^{M_{X, \max }} d M_{X} \frac{d \sigma(p p \rightarrow V X ; s)}{d y d M_{X}},
$$



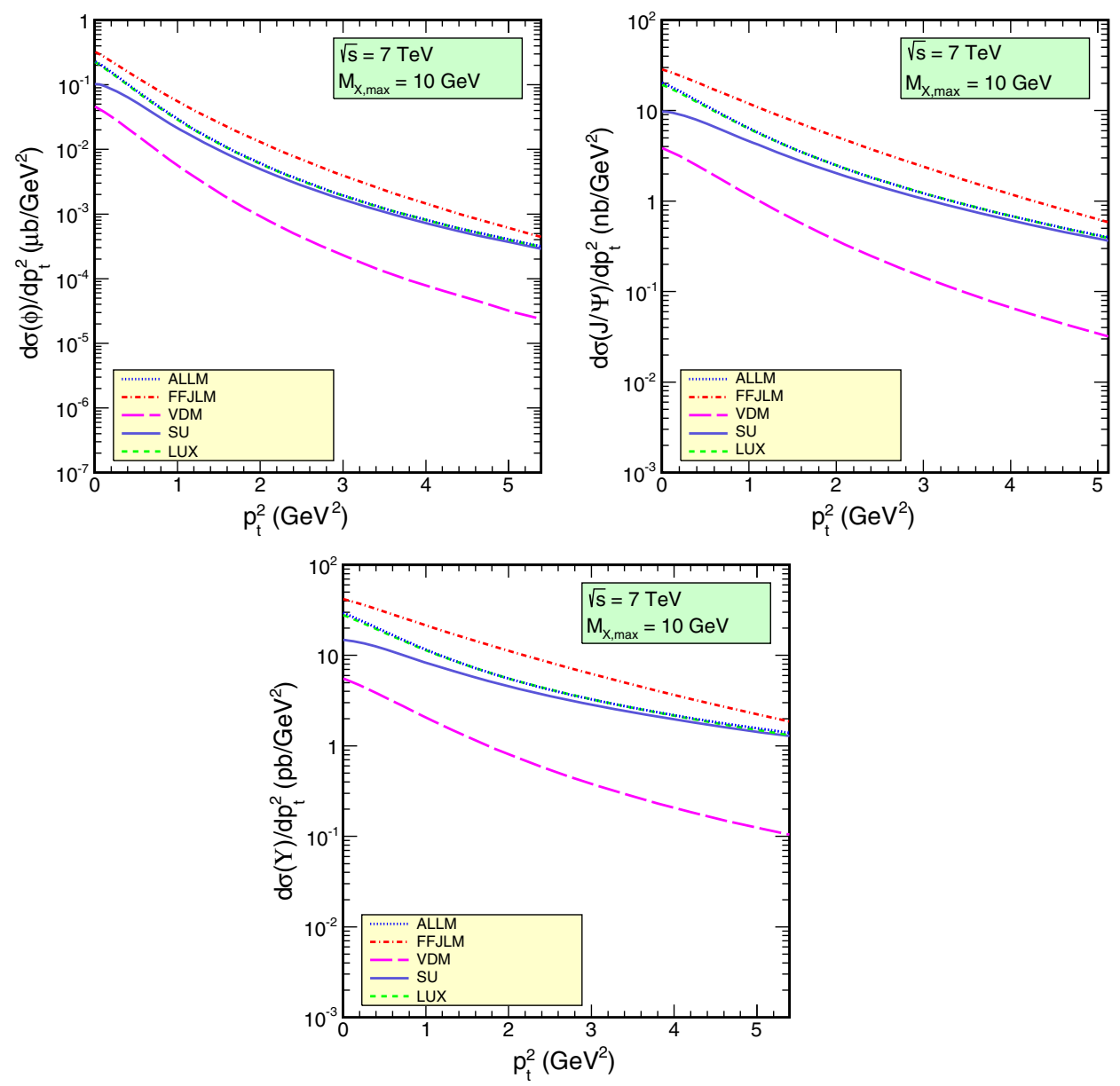

FIG. 7. Transverse momentum distribution of vector meson for $\mathrm{pp} \mathrm{cm}$ energy $\sqrt{s}=7 \mathrm{TeV}$ for the production of $\phi, J / \psi$, and $\Upsilon$ mesons for different parametrizations of the proton structure function $F_{2}$.

for $V=\phi, J / \psi, \Upsilon$ and $M_{X, \max }=10 \mathrm{GeV}$, using different parametrizations of the proton structure function $F_{2}$. For all of the used parametrizations of $F_{2}$, the rapidity distributions become narrower the heavier the produced meson. Let us briefly discuss the magnitude of the cross section predicted by the different parametrizations. We observe a very good agreement between the predictions using the ALLM and LUX-like parametrization. The results using the SU parametrization for the case of $\phi$ production differ somewhat in magnitude and shape from the results using previous two distributions, while for the case of $J / \psi$ and $\Upsilon$, the shapes of the rapidity distribution are similar, but there is still a $\sim 30 \%$ difference in the magnitude. We show separately the vector-dominance model (VDM) component of the SU parametrization. The FFJLM parametrization gives higher results than all other parametrizations. Notice however, that it is intended only for the region $M_{X} \lesssim 2 \mathrm{GeV}$.

In Figs. 7 and 8, we show the distributions in transverse momentum squared of the meson. Again, we integrate up to $M_{X, \max }=10 \mathrm{GeV}$,

$$
\frac{d \sigma(V)}{d p_{t}^{2}} \equiv \int_{M_{\mathrm{thr}}}^{M_{X, \max }} d M_{X} \frac{d \sigma(p p \rightarrow V X ; s)}{d p_{t}^{2} d M_{X}} .
$$

Regarding the different parametrizations of $F_{2}$, the transverse momentum distributions show a similar systematics as the rapidity distributions discussed above. Notice that the ALLM and LUX-like results basically coincide. We see that up to $p_{t}^{2} \lesssim 2 \mathrm{GeV}^{2}$ the $p_{t}^{2}$ distributions have an approximate exponential behavior $\propto \exp \left[-B_{\text {inel }} p_{t}^{2}\right]$.

In Fig. 9, we show the distribution in the invariant mass $M_{X}$ of the excited system. Here we see that the Fiore-fit behaves very differently from the other parametrizations at $M_{X}>2 \mathrm{GeV}$. Due to this unphysical behavior, it cannot be used for large $M_{X}$. On the other hand, the SzczurekUleshchenko parametrization appears to underestimate the cross section in the resonance region of small $M_{X}$. Here, the LUX-type fit, ALLM, and the Fiore parametrizations agree quite well.

Let us have a closer look at the correlation of the $M_{X}$ dependence with rapidity of the vector meson. In Fig. 10, we show the rapidity distribution of mesons for ALLM 

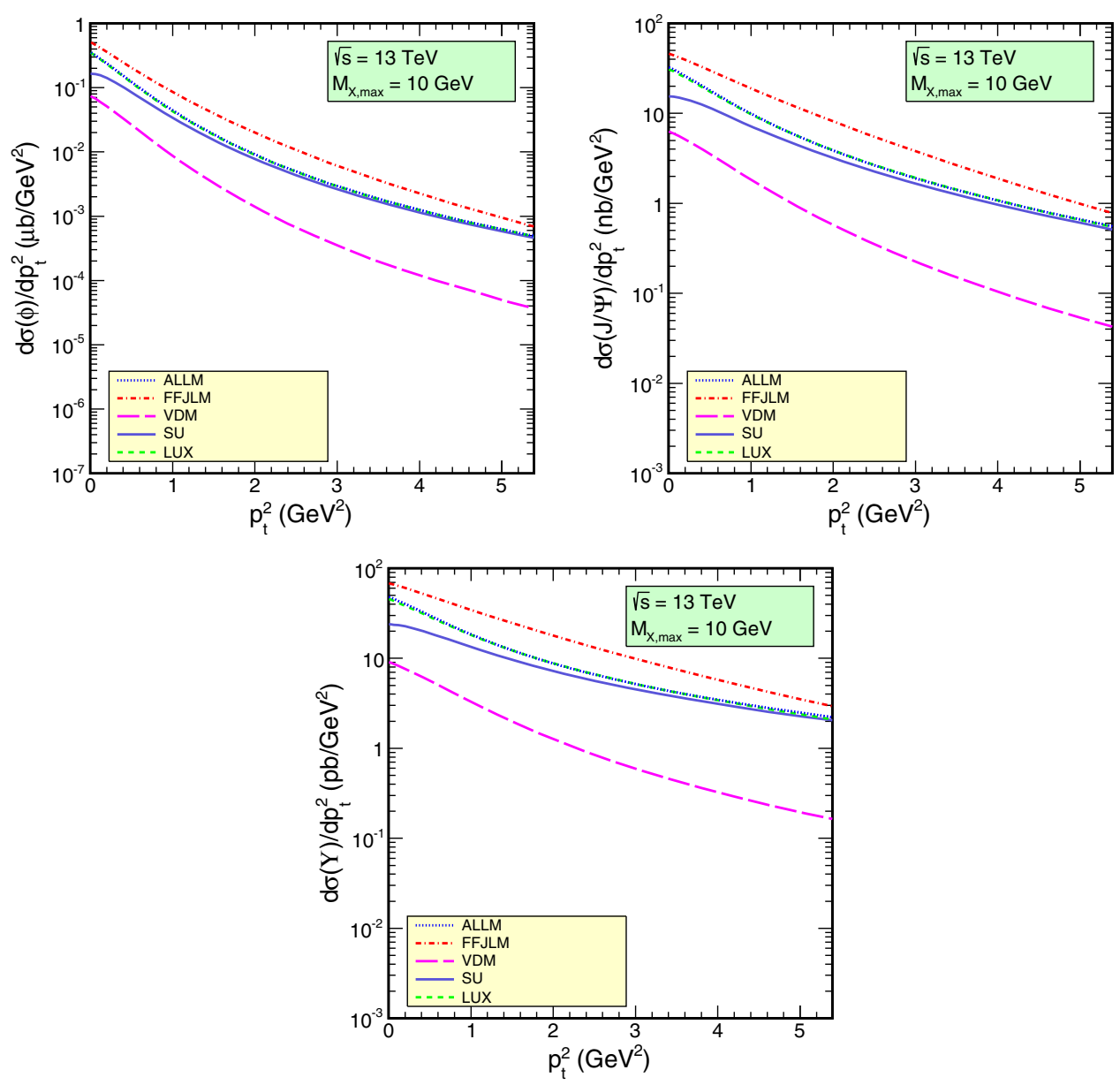

FIG. 8. Transverse momentum distribution of vector meson for pp $\mathrm{cm}$ energy $\sqrt{s}=13 \mathrm{TeV}$ for the production of $\phi, J / \psi$, and $\Upsilon$ mesons for different parametrizations of the proton structure function $F_{2}$.

structure function of a proton and different bins of $M_{X} \in\left[M_{X, \min }, M_{X, \max }\right]$. We observe that rapidity distributions for bins with $M_{X}>10 \mathrm{GeV}$ are peaked at midrapidity and are somewhat narrower than the contribution of $M_{X} \leq 10 \mathrm{GeV}$.

In Fig. 11, we show the rapidity distribution of mesons for different bins of $M_{X}$ for the FFJLM structure function of a proton, similarly as Fig. 10.

We return to the transverse momentum distributions in Fig. 12, where we plot the $p_{t}^{2}$ distribution with $M_{X}$ integrated up to different values of $M_{X \text {,max }}$. We observe that the contribution from the resonance region of $M_{X}<$ $2 \mathrm{GeV}$ has a much softer tail at large $p_{t}^{2}$ than when the large-mass contribution is added. For comparison, we also show the $p_{t}^{2}$ distribution of the exclusive $p p \rightarrow$ $p p V$ contribution [1]. The theoretical analysis for exclusive photoproduction in proton-proton collisions was shown also in papers [24-26]. The shape of the $p_{t}^{2}$ distributions only weakly depends on energy.

Under the conditions of LHC experiments, like $[2,3]$, the dissociative diffractive production is a background to the fully exclusive production with intact protons.
In order to highlight the difference of the inelastic contribution and the exclusive one, we introduce the ratios,

$$
\begin{aligned}
R^{\mathrm{EM} / \operatorname{excl}}\left(p_{t}, M_{X, \text { max }}\right) & =\frac{\int_{M_{\mathrm{thr}}}^{M_{X, \max }} d M_{X} \frac{d \sigma(p p \rightarrow p V X)}{d p_{t} d M_{X}}}{\frac{d \sigma(p p \rightarrow p V p)}{d p_{t}}}, \\
R^{\mathrm{EM} / \operatorname{excl}}\left(y, M_{X, \text { max }}\right) & =\frac{\int_{M_{\mathrm{thr}}}^{M_{X, \max }} d M_{X} \frac{d \sigma(p p \rightarrow p V X)}{d y d M_{X}}}{\frac{d \sigma(p p \rightarrow p V p)}{d y}} .
\end{aligned}
$$

In Fig. 13, we show the ratio $R^{\mathrm{EM} / \mathrm{excl}}$ as a function of $p_{t}$ for different upper limits on $M_{X}$; we see that as soon high mass states are included, the inelastic contribution dominates at $p_{t} \gtrsim 1 \mathrm{GeV}$. In Fig. 14, we have a closer look at $R^{\mathrm{EM} / \mathrm{excl}}$ for the excitation of low-mass states $M_{X}<2 \mathrm{GeV}$. Here, we use both the ALLM and Fiore parametrizations. We see that for $\phi$ production the ratio is always smaller than one, while for heavier mesons, inelastic production will dominate at $p_{t} \gtrsim 1.5 \mathrm{GeV}$.

In Figs. 15 and 16, we show the analogous ratios for the rapidity distribution. 

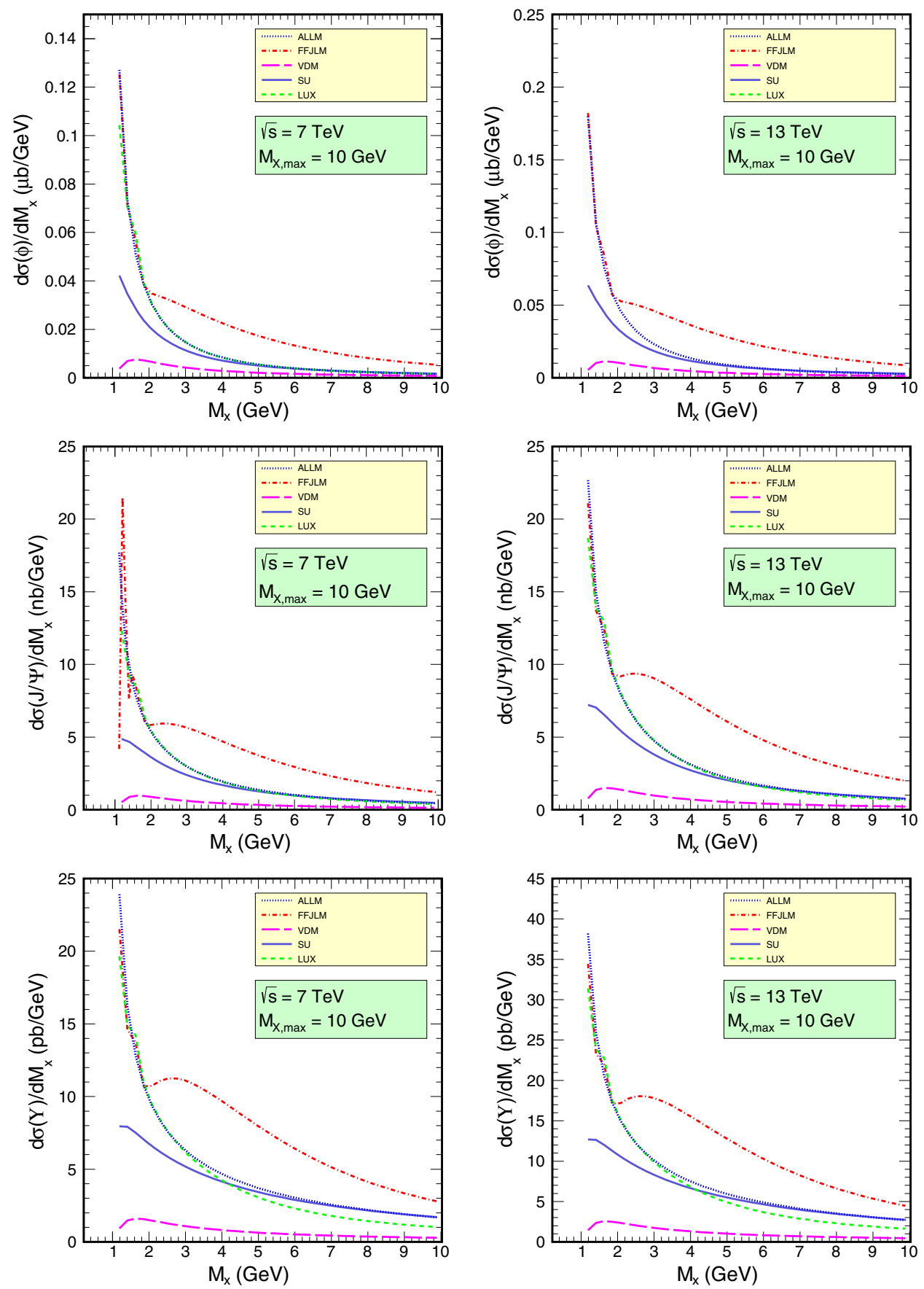

FIG. 9. Distribution of the invariant mass $M_{X}$ of the excited system for the pp cm energy $\sqrt{s}=7 \mathrm{TeV}$ (left panels) and $\sqrt{s}=13 \mathrm{TeV}$ (right panels). 

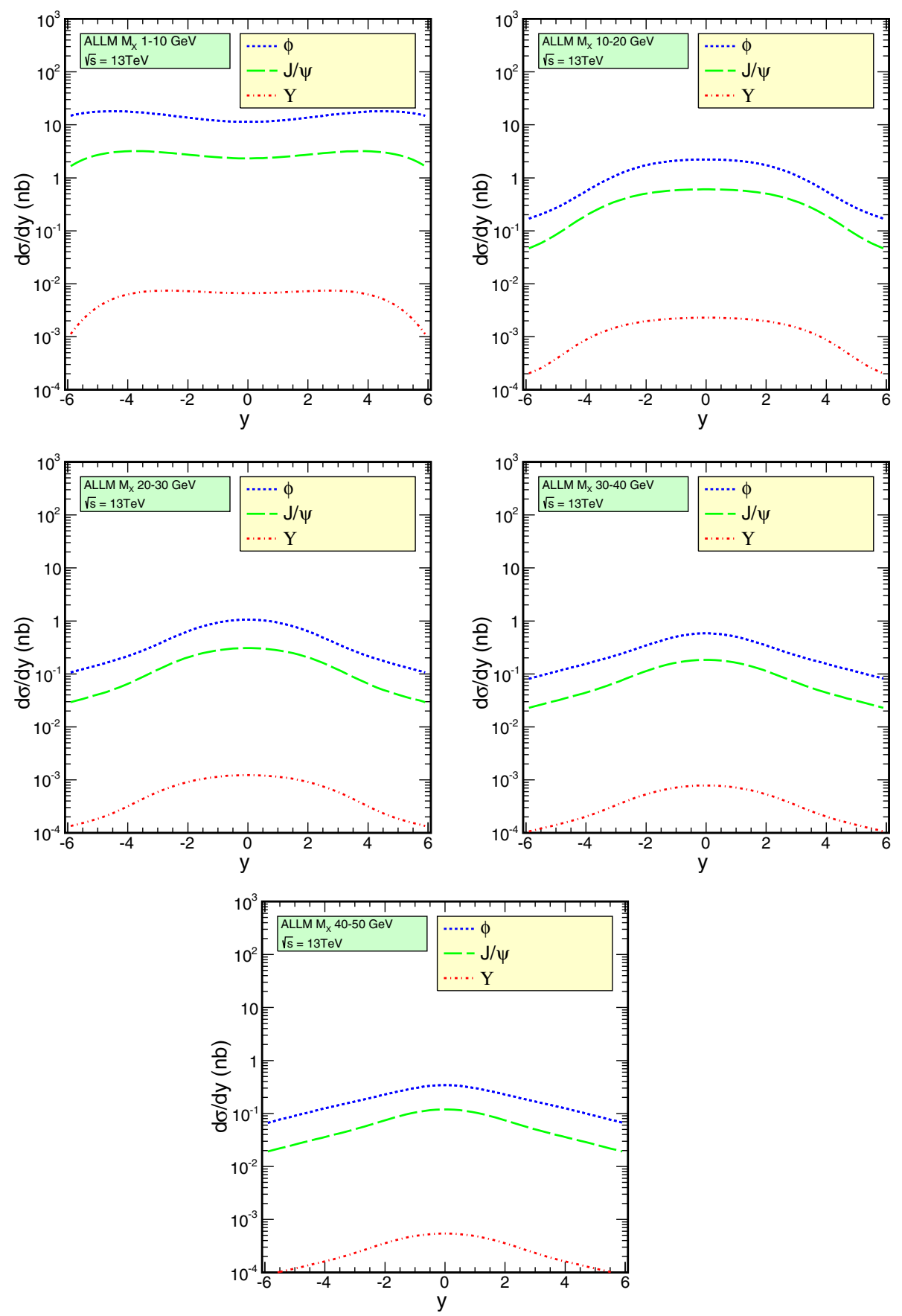

FIG. 10. Rapidity distribution of vector mesons for different bins of the invariant mass of the excited system for the ALLM parametrization. 

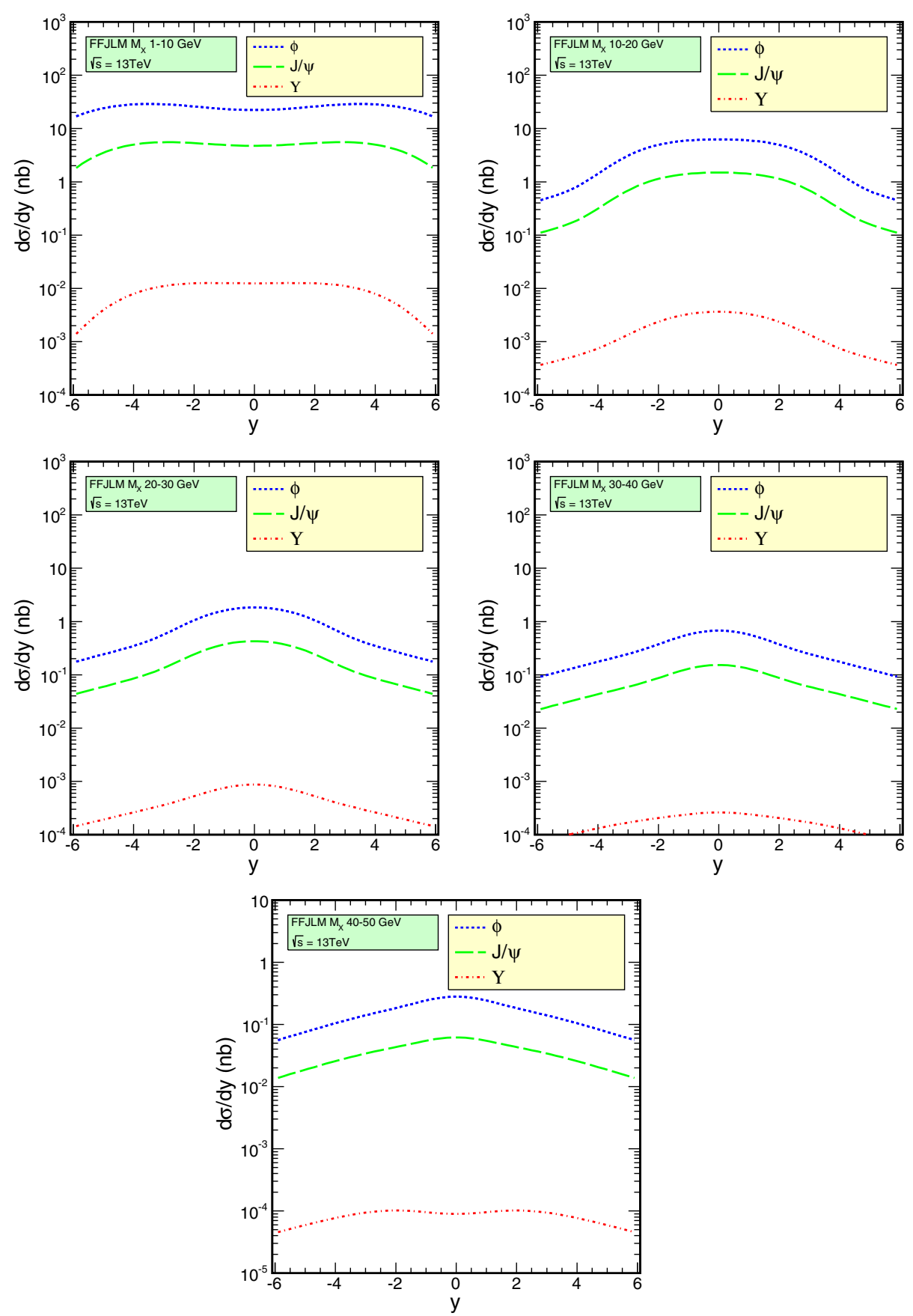

FIG. 11. Rapidity distribution of vector mesons for different bins of the invariant mass of the excited system for the FFJLM parametrization. 

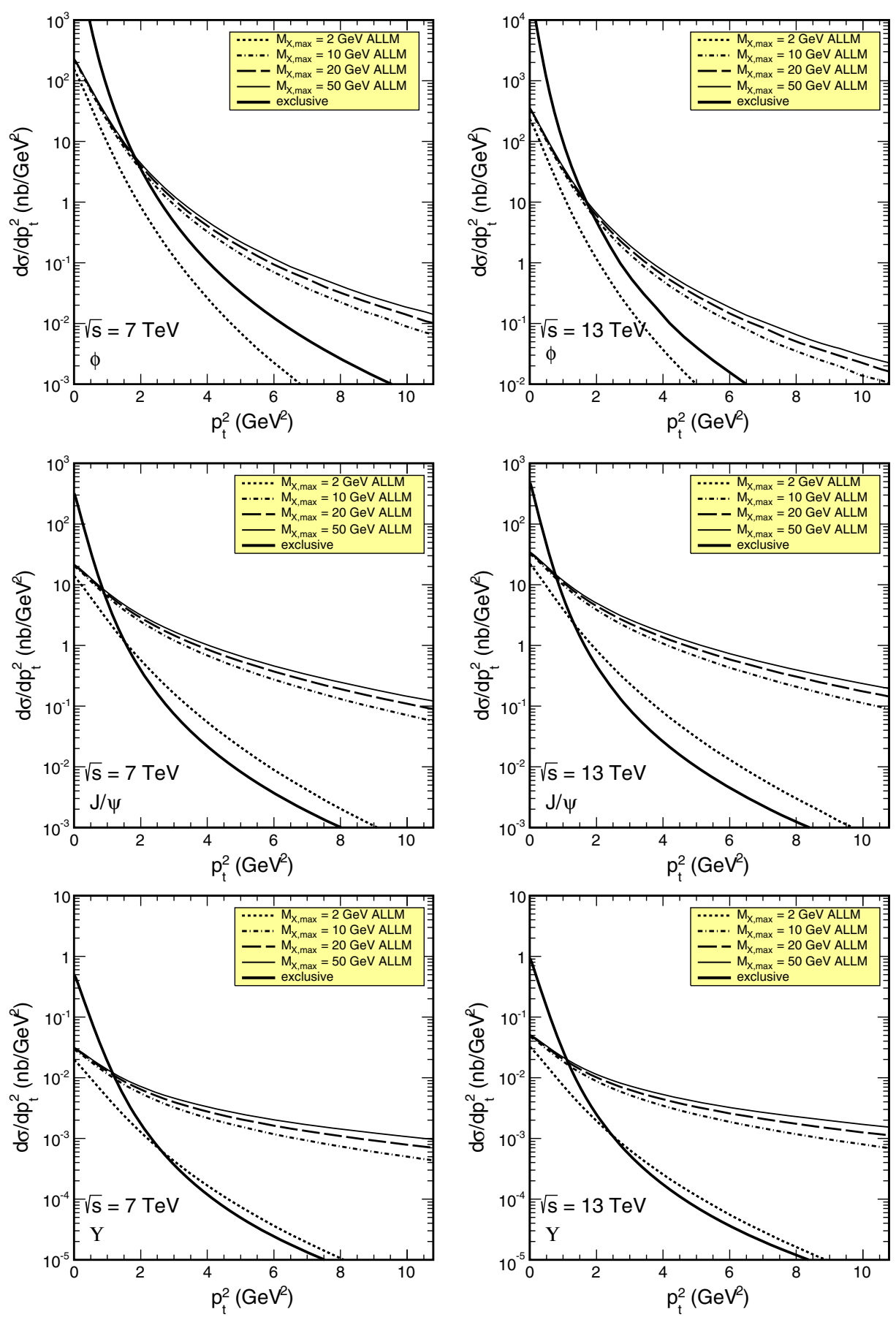

FIG. 12. $p_{t}^{2}$-distribution of vector mesons for different upper limits on the invariant mass of the excited system. 

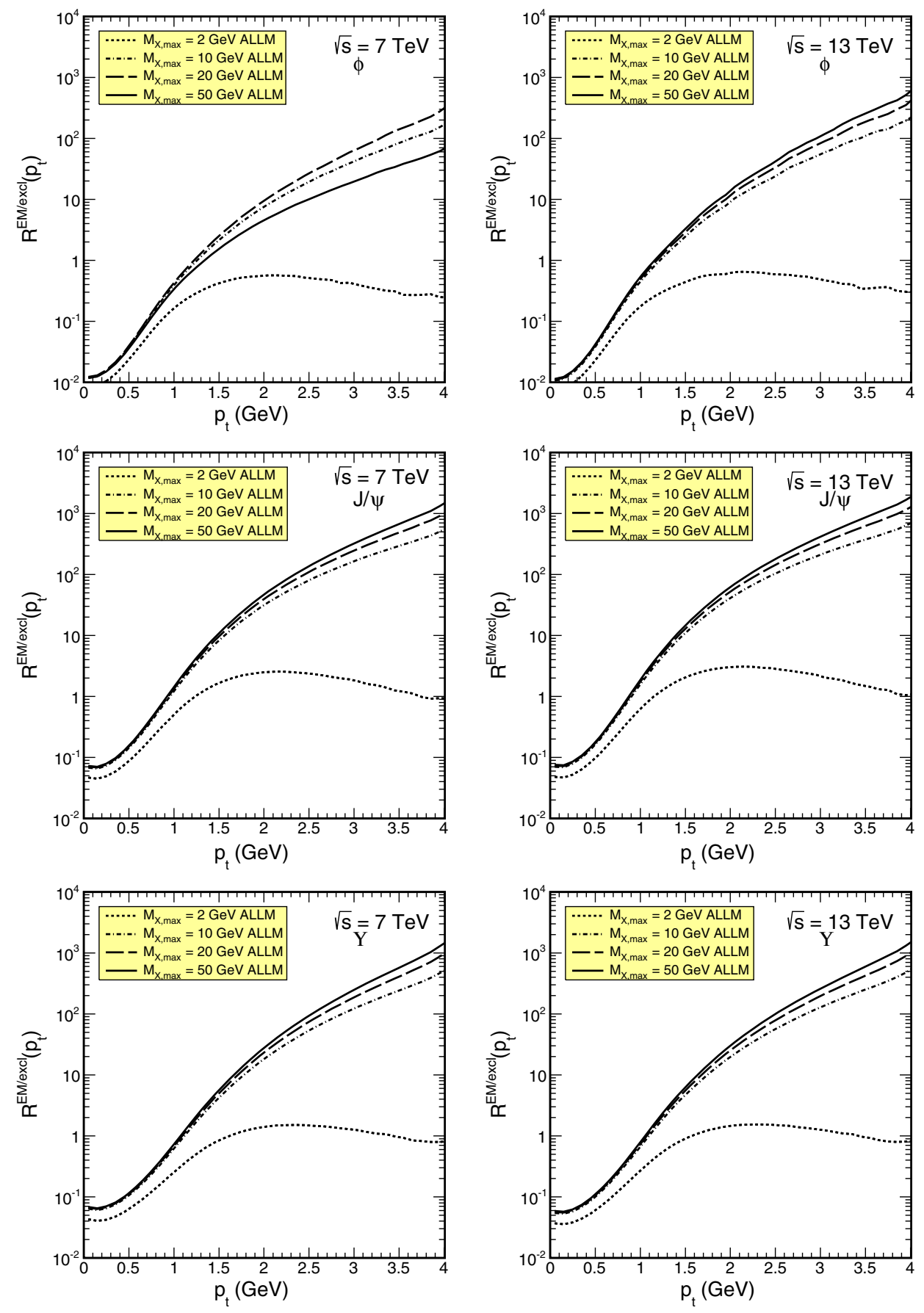

FIG. 13. Ratio of inelastic diffractive to exclusive vector meson production as a function of transverse momentum for different upper limits on the excited mass $M_{X}$. 

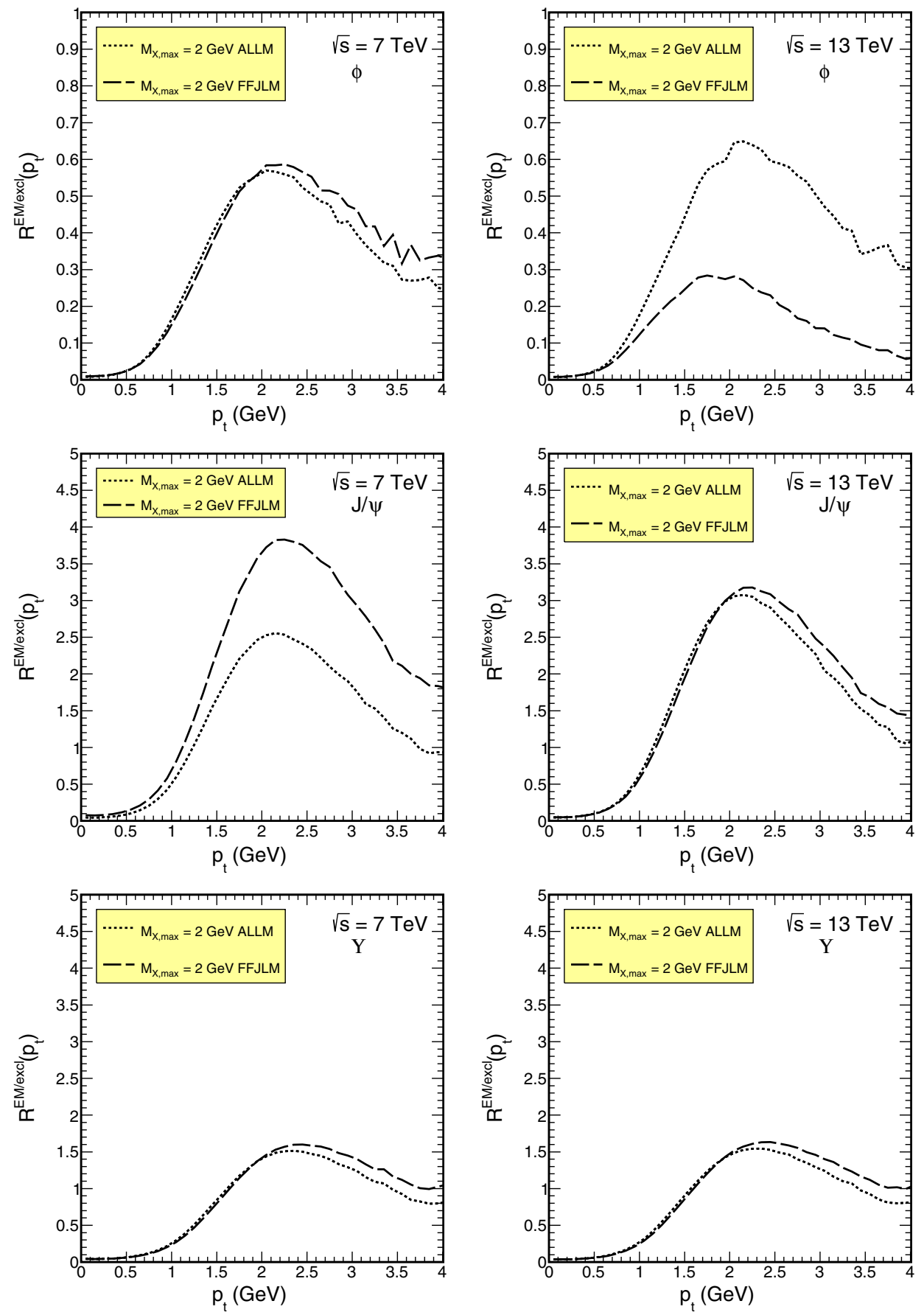

FIG. 14. Ratio of inelastic diffractive to exclusive vector meson production as a function of transverse momentum for low proton excited masses, integrated up to $M_{X}=2 \mathrm{GeV}$. 

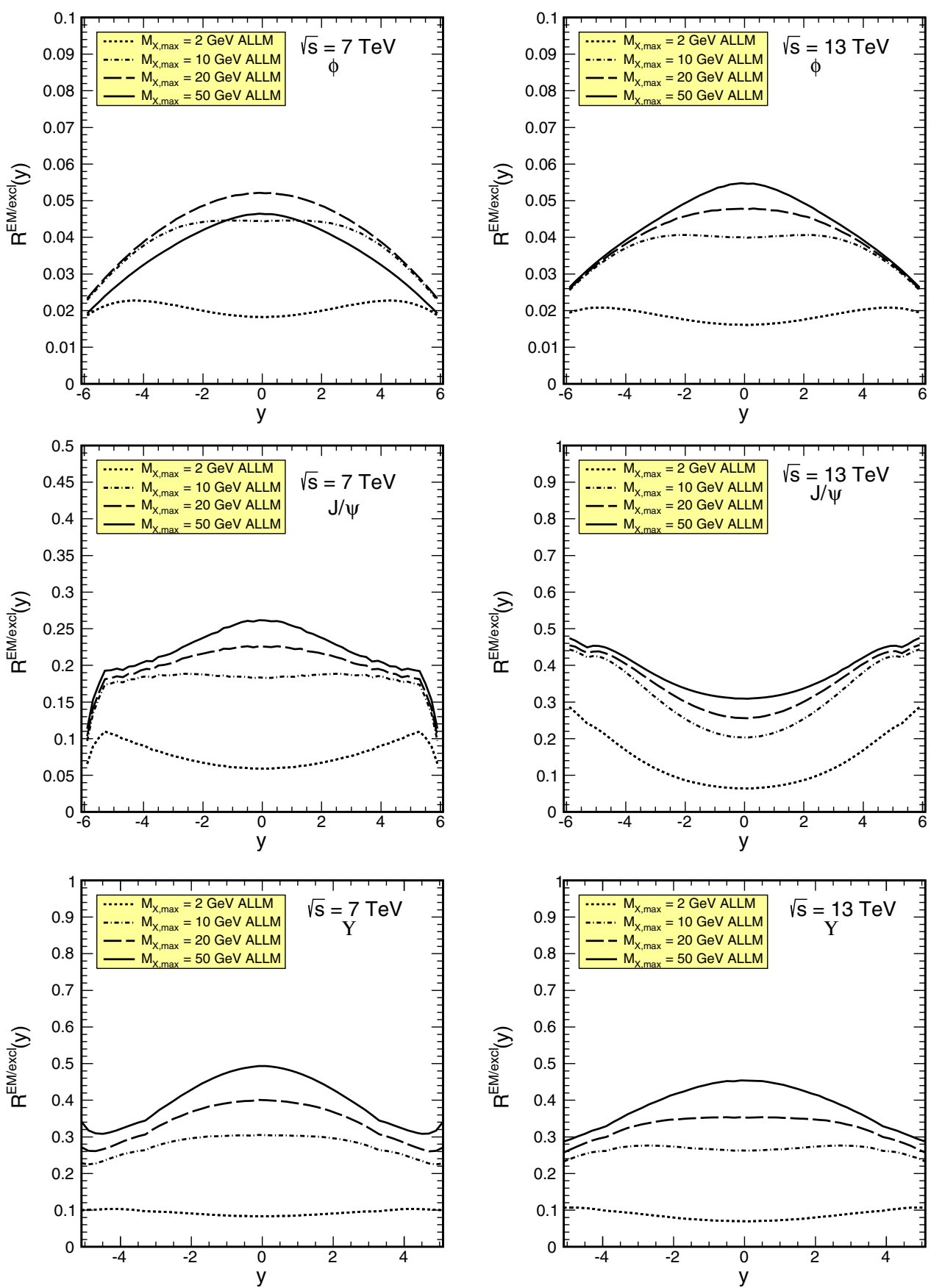

FIG. 15. Ratio of inelastic diffractive to exclusive vector meson production as a function of rapidity for different upper limits on the excited mass $M_{X}$. 

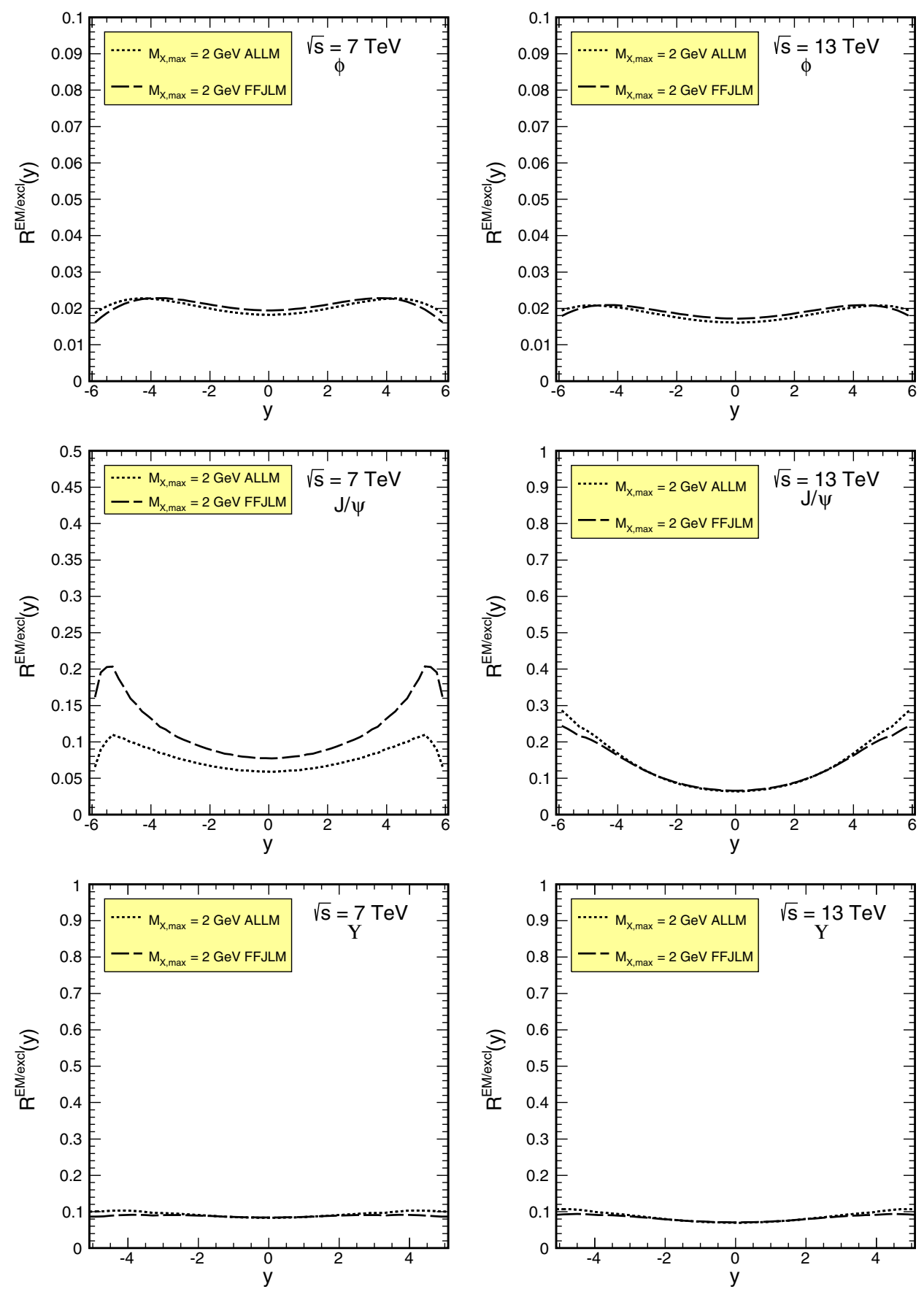

FIG. 16. Ratio of inelastic diffractive to exclusive vector meson production as a function of rapidity for low excited masses, integrated up to $M_{X}=2 \mathrm{GeV}$. 


\section{CONCLUSIONS}

In this paper, we have discussed the semiexclusive production of vector mesons in $p p \rightarrow V p X$ processes via photon-pomeron or pomeron-proton fusion, where $X$ stands for an excited/dissociated proton system and $V=\phi, J / \psi, \Upsilon$. We have investigated the similarities and differences of various cross sections to the exclusive $p p \rightarrow p p V$ process. The electromagnetic dissociation of protons is calculated using an inelastic unintegrated photon flux, which was calculated based on modern parametrizations of deep-inelastic proton structure functions. Different parametrizations from the literature have been used.

A number of differential cross distributions for the vector mesons (in their rapidity and transverse momentum) as well as for the mass of the dissociative system, remnant of the proton, have been calculated. We have found that in all the considered cases the photon dissociation cross section is large compared to its purely exclusive counterpart. The results strongly depend on the parametrization of the structure function used. One should stress, however, in this context that different parametrizations have quite different status. Some of them are global fits to the world data, often not ideal in some other regions of the phase space. Some of them focus rather on some corners of the phase space, so are extremely good there. However, they can be not realistic in other corners of the phase space. We have discussed in detail which distributions provide realistic estimates of the cross section.

The semiexclusive contributions produce vector mesons with large transverse momenta. The rapidity distributions of semiexclusive and purely exclusive distributions are rather similar. In the present analysis, we have shown also the ratio of the semiexclusive to the purely exclusive contributions. This ratio depends strongly on the vector meson transverse momentum and only mildly on rapidity. In general, a bigger ratio is obtained for heavier quarkonia.

It is obvious that a large fraction of the remnant cannot be seen by central detectors of different LHC experiments in the case when protons are not measured using specially dedicated forward detectors, just installed recently by the CMS-TOTEM or ATLAS Collaborations. Without measuring both protons, as is the case of the LHCb experiment, the so-called exclusive data are not fully exclusive and may contain the semiexclusive contributions discussed here. The LHCb Collaboration cuts the large- $p_{t}$ part of the corresponding distribution in a purely phenomenological fit of different slope. It is not completely clear how good is such a procedure. It would be good to relax requirements on rapidity gap(s) around vector mesons and actually measure the semieexclusive contributions. We encourage experimentalists to perform such analyses. We note that the semiexclusive contributions were not measure so far, but are interesting themselves. Such measurements would be therefore tests of the method used here.

\section{ACKNOWLEDGMENTS}

This work was partially supported by the Polish National Science Center Grant No. UMO-2018/31BST2/03537 and by the Center for Innovation and Transfer of Natural Sciences and Engineering Knowledge in Rzeszów.
[1] A. Cisek, W. Schäfer, and A. Szczurek, J. High Energy Phys. 04 (2015) 159.

[2] R. Aaij et al. (LHCb Collaboration), J. Phys. G 40, 045001 (2013).

[3] R. Aaij et al. (LHCb Collaboration), J. High Energy Phys. 09 (2015) 084.

[4] A. Cisek, W. Schäfer, and A. Szczurek, Phys. Lett. B 769, 176 (2017).

[5] R. Fiore, A. Flachi, L. L. Jenkovszky, A. I. Lengyel, and V. K. Magas, Eur. Phys. J. A 15, 505 (2002).

[6] R. Fiore, L. L. Jenkovszky, F. Paccanoni, and A. Prokudin, Phys. Rev. D 70, 054003 (2004).

[7] H. Abramowicz, E. M. Levin, A. Levy, and U. Maor, Phys. Lett. B 269, 465 (1991).

[8] H. Abramowicz and A. Levy, arXiv:hep-ph/9712415.

[9] M. Łuszczak, W. Schäfer, and A. Szczurek, Phys. Rev. D 93, 074018 (2016).

[10] A. D. Martin, W. J. Stirling, R. S. Thorne, and G. Watt, Eur. Phys. J. C 63, 189 (2009).
[11] P. E. Bosted and M.E. Christy, Phys. Rev. C 77, 065206 (2008).

[12] A. Airapetian et al. (HERMES Collaboration), J. High Energy Phys. 05 (2011) 126.

[13] K. Abe et al. (E143 Collaboration), Phys. Lett. B 452, 194 (1999).

[14] A. Manohar, P. Nason, G. P. Salam, and G. Zanderighi, Phys. Rev. Lett. 117, 242002 (2016).

[15] A. V. Manohar, P. Nason, G. P. Salam, and G. Zanderighi, J. High Energy Phys. 12 (2017) 046.

[16] A. Szczurek and V. Uleshchenko, Eur. Phys. C 12, 663 (2000); Phys. Lett. B 475, 120 (2000).

[17] I. P. Ivanov, N. N. Nikolaev, and A. A. Savin, Phys. Part. Nucl. 37, 1 (2006).

[18] A. Cisek, W. Schäfer, and A. Szczurek, Phys. Lett. B 690, 168 (2010).

[19] K. Kutak and A. M. Stasto, Eur. Phys. J. C 41, 343 (2005).

[20] C. Alexa et al. (H1 Collaboration), Eur. Phys. J. C 73, 2466 (2013). 
[21] A. Aktas et al. (H1 Collaboration), Phys. Lett. B 568, 205 (2003).

[22] W. Schäfer and A. Szczurek, Phys. Rev. D 76, 094014 (2007).

[23] M. Derrick et al. (ZEUS Collaboration), Phys. Lett. B 377, 259 (1996).
[24] L. Motyka and G. Watt, Phys. Rev. D 78, 014023 (2008).

[25] S. P. Jones, A. D. Martin, M. G. Ryskin, and T. Teubner, J. High Energy Phys. 11 (2013) 085.

[26] V. P. Goncalves, L. A. S. Martins, and W. K. Sauter, Eur. Phys. J. C 76, 97 (2016). 